\title{
Geolocation Spectrum Database Assisted Optimal Power Allocation: Device-to-Device Communications in TV White Space
}

\author{
Zhen Xue, Liang Shen, Guoru Ding, Qihui Wu \\ College of Communications Engineering, \\ PLA University of Science and Technology, Nanjing 210007, China \\ [e-mail: 15895872631@yeah.net] \\ *Corresponding author: Guoru Ding \\ Received December 8, 2014; revised September 19, 2015; accepted October 13, 2015; \\ published December 31, 2015
}

\begin{abstract}
TV white space (TVWS) is showing promise to become the first widespread practical application of cognitive technology. In fact, regulators worldwide are beginning to allow access to the TV band for secondary users, on the provision that they access the geolocation database. Device-to-device (D2D) can improve the spectrum efficiency, but large-scale D2D communications that underlie TVWS may generate undesirable interference to TV receivers and cause severe mutual interference. In this paper, we use an established geolocation database to investigate the power allocation problem, in order to maximize the total sum throughput of D2D links in TVWS while guaranteeing the quality-of-service (QoS) requirement for both D2D links and TV receivers. Firstly, we formulate an optimization problem based on the system model, which is nonconvex and intractable. Secondly, we use an effective approach to convert the original problem into a series of convex problems and we solve these problems using interior point methods that have polynomial computational complexity. Additionally, we propose an iterative algorithm based on the barrier method to locate the optimal solution. Simulation results show that the proposed algorithm has strong performance with high approximation accuracy for both small and large dimensional problems, and it is superior to both the active set algorithm and genetic algorithm.
\end{abstract}

Keywords: TV white space, geolocation spectrum database, D2D, spatial reuse, optimal power allocation, convex optimization

A preliminary version of this paper appeared in IEEE ICC 2009, June 14-18, Dresden, Germany. This version includes a concrete analysis and supporting implementation results on MICAz sensor nodes. This research was supported by a research grant from the IT R\&D program of MKE/IITA, the Korean government [2005-Y-001-04, Development of Next Generation Security Technology]. We express our thanks to Dr. Richard Berke who checked our manuscript. 


\section{Introduction}

$\mathbf{I}_{\mathrm{n}}$ the past decade, wireless communications have developed rapidly. There are increasing numbers of wireless devices that are used in modern society. The wireless spectrum is scarce and limitations on useable resources are hindering the evolution of modern wireless communication systems [1]. Cognitive radio (CR) is a feasible technology that can provide a secondary use for TV white space (TVWS), which is being intensively researched [2]. It is worth mentioning that IEEE 802.22 is the driver in advancing CR towards practical use. This standard allows wireless regional area networks (WRANs) to utilize the TV bands to offer broadband service. The emergence of a geolocation spectrum database is a promising cognitive technique which can enable TVWS to be used for wireless communication, especially after the FCC ruling [3] in 2010, which removed the requirement for mandatory spectrum sensing in white space. Instead, the white space device (WSD) should learn spectrum availability at its current location from an authorized database [4].

Unlicensed users can utilize TVWS only if they can guarantee protection to neighboring incumbent networks. In the ECC report 159 [5], a deterministic approach called 'Reference Geometry' was proposed. In contrast with previous works, this aimed to address not only co-channel interference (CCI) but also adjacent channel interference (ACI) at the TV receiver. In [6], the authors proposed an analytical approach to determine the permissible transmission power for short-range secondary users under aggregate adjacent channel interference constraints in TV white space. A hybrid spectrum sensing and geolocation database framework is proposed in [7] to fully locate available TVWS, which serves as a fundamental framework for advancing the design of hybrid approaches for spatial-temporal spectrum hole discovery. These works mainly focus on protecting the TV receiver, rather than considering secondary users.

Since there are plenty of opportunities for spatial reuse, we integrate device-to-device communication into TVWS to explore the unused TV spectrum. The benefits of D2D communications are manifold: an increased network capacity, higher transmission rate, ability to offload data and creation of new applications [8, 9]. Cellular topology is usually adopted in WRAN/WLAN communications to manage the networks, however this has two crucial constraints [10]. One is the limited network capacity, which is due to many reasons including the fact that only a single pair of transceivers can communicate on a channel simultaneously, without exploiting potential opportunities for spatial reuse of channels. Another drawback is energy efficiency, which is important since the user device may have energy limitations, and there will be an increase in energy requirements when devices are far from the base station (BS) or the communication links are poor condition. In WRAN/WLAN networks, multiple adjacent TV channels can be used as a bundled channel, however non-adjacent channels cannot be used at the same time in a cell, and as a result vacant channels may be wasted. All of those constraints can be avoided by direct device-to-device communication, which saves unnecessary routing through a BS when mobile devices need proximity-based services, and hence decreases the end-to-end delay. There has recently been tremendous interest in supporting D2D communication, driven largely by social networking applications. In [11], a novel hierarchical cloud computing architecture is designed to enhance performance by offloading Long Term Evolution-Advanced (LTE-A) to D2D communication. The proposed architecture can increase the capacity of a mobile network by up to 10 percent. The Third Generation Partnership Project (3GPP) is attempting to incorporate D2D into LTE to support 
public safety networks [12]. Moreover, the IEEE 802.22b Task Group has been established to enhance the WRAN's capacity in TV bands by D2D communication and multiple-input multiple-output (MIMO) [10].

There are various schemes that have been proposed to manage interference in D2D communications. [13] has investigated the optimal method of power control along with mode switching with respect to the overall network throughput, however only a prioritized user is given a minimum service guarantee. In [14], a fixed power margin scheme is proposed to coordinate interference between D2D pairs and licensed users. This scheme is simple but not optimal since it is difficult to select a suitable power margin. To manage interference between cellular users and D2D systems, [15] has proposed an interference limited area (ILA) control scheme, which disallows the coexistence of cellular users and a D2D pair within a defined area. Specifically, we investigate the problem of D2D underlying TVWS using code-division multiple access (CDMA) technology with explicit interference protection for incumbent TV receivers and QoS constraints for multiple D2D pairs. We assume that the mobile user/device in each D2D link also requires a minimum transmission rate in terms of signal to interference plus noise ratio (SINR) and impose maximum power constraints. There are some studies $[8,16]$ focusing on guaranteeing quality-of-service (QoS) requirements for both D2D users and regular cellular users, but [16] has only addressed the case where one D2D pair reuses resource with a cellular user. However, the number of D2D users is relatively small with respect to existing cellular users and the number of potentially available channels in these studies. In this paper, we are attempting to apply large numbers of D2D links in TVWS. Similar to cellular networks, D2D systems in TVWS also need a central controller. The difference is that although D2D users do not experience congestion from cellular users, they are responsible for protecting nearby TV receivers.

The deployment of multiple D2D links in TVWS poses a critical problem, which is the coexistence between TV receivers and D2D links, as well as self-coexistence between multiple D2D links. We study an optimization problem to maximize the total sum throughput of D2D links under the constraint of coexistent conditions. Since this type of optimization problem is nonconvex, it is difficult to calculate a global optimal solution. Based on these technical challenges, our main contributions can be summarized as follows:

- Present a novel network scenario and framework within multiple D2D communications underlying TVWS. In this system, D2D links are regulated by the access point, and the dominant feature is the geolocation database. New users are registered at the database through AP and the database uses algorithms to allocate power for each link from an overall perspective.

- Formulate the spatial reuse of TV channels between licensed DTV services and unlicensed D2D communications as an optimization problem. The objective is the total sum throughput of the D2D links. The constraint conditions are the QoS requirements for both the compromised TV receiver and each D2D link, and the maximum transmission power of the devices. This optimization problem is challenging, but we take an effective approach to convert it into a series of tractable convex sub-problems, making it possible to design an effective algorithm to solve it.

- Propose a barrier method based algorithm. We first explored the existence of an optimal solution with respect to a feasible system, and used the barrier method to locate the optimum value in polynomial complexity. It has been proven that the algorithm converges well and provides a high approximation accuracy of the original objective. Additionally, the proposed algorithm outperforms both the active set algorithm and the genetic algorithm. 


\section{System Model Description}

\subsection{Network Scenario}

As shown in Fig. 1, the white space device (WSD) should transmit its signal by opportunistically utilizing TVWS in order to avoid causing harmful interference to licensed DTV signal receivers. In the near future, a WSD will be more similar to a personal device such as smart phone, tablet or other device equipped with programmable support hardware. In the case of a concert or an athletic competition, there are a large number of users/devices willing to share audio or video materials with others nearby, which may cause a high traffic load for the cellular network. However, D2D communications can provide an efficient solution for these types of proximity-based media services. Since many mobile users/devices are accessing the licensed spectrum at the same time, this may result in unacceptable interference levels in the primary service. Unlicensed spectrums such as the TV band offer a better choice for D2D communications at lower expense. An access point and a low-cost infrastructure is needed to coordinate those devices. Usually, an AP is not necessary for a new infrastructure since cellular infrastructure is already widely deployed. It can be a very promising business model for the cellular service provider to exploit TVWS and offer additional spectrum to mobile users/devices [17]. There is one more deployment to implement D2D communications in TVWS, which is that the AP should be connected to a well-established geolocation spectrum database, which includes overall and reliability spectrum availability information to any particular area of interest.

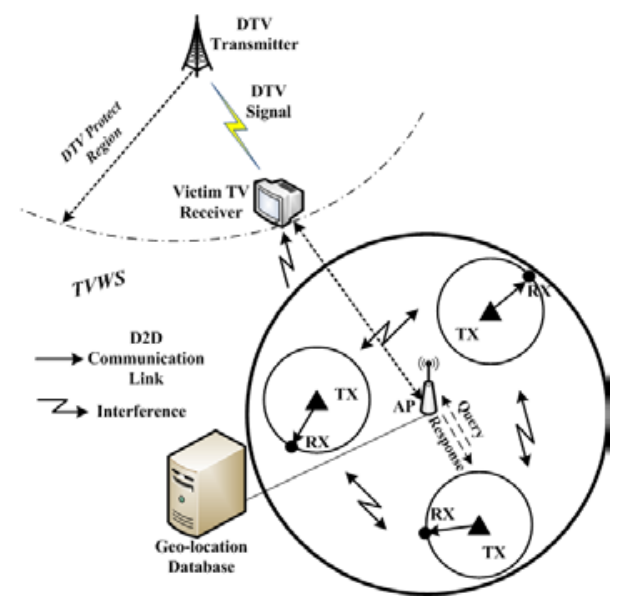

Fig. 1. Scenario of D2D communications in TVWS

In Fig. 1, broken lines with an arrow represent interference. Individual D2D links have mutual interference between them and multiple mobile users/devices can cause cumulative interference to the victim TV receiver. A solid arrow represents the desired signal. The AP takes control of the D2D links via an exclusive control channel which must not be occupied by any communication links. This can be realized by broadcasting a beacon signal periodically. The active D2D links listen to the control beacons and follow the control information [18].

As a direct transmission technique, it is critical for users/devices within the proximity to find each other, which is called D2D peer device discovery [8]. Devices then must establish D2D sessions to maintain continuous network management and control for the potential data transmission [19]. However, the most challenging and important issue in D2D communications is efficient resource allocation and guarantee of QoS. The process of D2D 
link access to TVWS is illustrated in Fig. 2 and shows that a single period contains three basic time slots: a communication request window, a session setup window and a communication window. Before starting communications, a WSD pair must inform the access point by initiating a query request within the time slot of a communication request window. To discover the target peer device, users/devices send packets to the AP via a commonly used cellular channel. Specifically, the information in the packet includes location information for determination of the available spectrum, the device ID and the target peer ID for establishment of the communication link, and the QoS requirement to guarantee effective signal receipt. The AP then accesses the central geolocation database, to get the available spectrum for users/devices based on the users/devices that are already registered in the geolocation database. Similar to the IP-based detection method to develop D2D session setup in [19], AP handles the whole procedure and the D2D session setup is transparent to the users/devices. In the session setup window, AP firstly responds to users/devices with the corresponding available spectrum in slot b1. In the next slot b2, users/devices then report the channel gain measurements to AP. In slot b3, the geolocation database executes the power allocation algorithm for the registered users/devices. After adjusting the transmission power based on feedback from the AP, the registered users/devices can start data transmission in the communication window. We assume all the time windows and slots have been well designed, taking both efficiency and overheads into consideration. At the beginning of a new period, users/devices can choose to evacuate or stay by informing the AP at slot $\mathrm{d}$, which is same as the procedure in slot a.

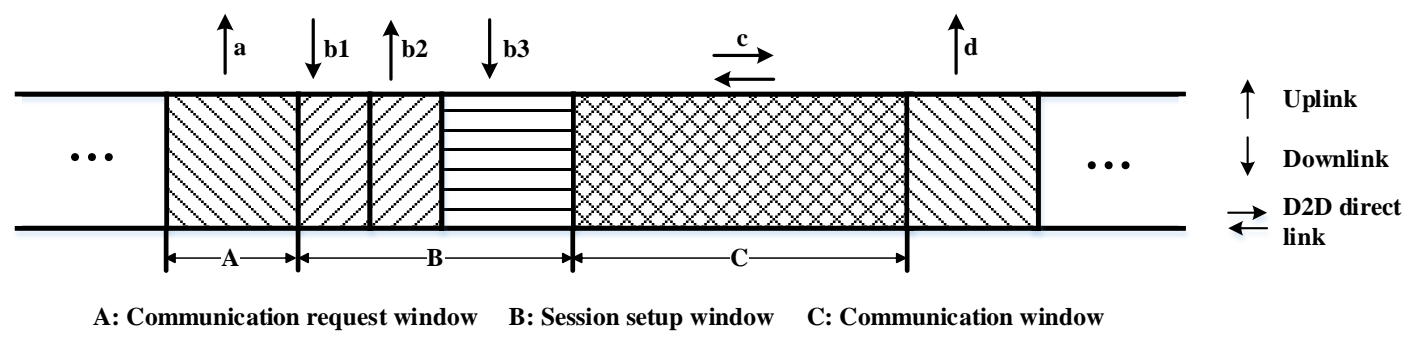

Fig. 2. The process of D2D access to TVWS

In a real scenario, there may be hundreds of active users making communication requests within a single slot. However, due to a limited available spectrum, the geolocation database cannot provide each user with exclusive use. Since different user pairs are distributed over a relatively wide range, there are many opportunities to exploit spatial reuse in each channel. In order to accommodate as many as possible user pairs with limited TV channels, we have previously proposed two spectrum assignment methods in [20]. Therefore, in this paper we emphasize power allocation to maximize the throughput performance for such a high volume of users. If there are too many active users to reuse the spatial spectrum, the admission control scheme must be activated, which may result in some user pairs not being permitted access, due to their poor SINR level or severe interference to others [21]. For any defined signal strength, the received interference strength directly affects the QoS of each user. Since there may be a high distribution density of mobile devices, mutual interference may arise from both the existing channel and adjacent channels. This is discussed further in Section 2.3.

To facilitate analysis in this paper, we choose a TV receiver that lies on the intersection of the DTV signal cover contour and the line from the DTV transmitter to the secondary cell center, which is the worst case scenario that will be confronted with the most aggregate interference [7]. We hereafter refer to this receiver as the victim TV receiver and give it 
adequate protection. In the rest of this section, we first introduce the propagation model that is used in this paper, and then illustrate the SINR threshold model.

\subsection{Signal Propagation Model}

Since all of the D2D links are within the communication area of a specific cell, the transmitter and receiver pair is assumed to be distributed around the AP, which is located at the center of a cell. In many previous studies, the signal propagation channel has been modeled based on two major dynamics: the large-scale distance-dependent path loss and the small-scale fading [22]. The following is used to evaluate the large-scale path loss between a transmitter and a receiver, which is the $\mathrm{dB}$ mode of $g_{r x, t x}$ [23],

$$
G_{r x, t x}[\mathrm{~dB}]=10 * \alpha \log (4 \pi d)+10 * \alpha \log (f)-20 \log \left(l_{T} * l_{R}\right),
$$

where $\alpha$ is the path loss exponent which depends on the specific propagation environment, $d$ is the distance from the transmitter to the receiver in $\mathrm{m}, f$ is the central frequency in $\mathrm{MHz}$, and $l_{T}$ and $l_{R}$ are the height of the transmitter and the receiver respectively in $\mathrm{m}$.

Small-scale fast fading cannot be precisely predicted using current models. This quantity is usually characterized by a probability distribution (e.g., Rayleigh, Rice or Nakagami). We use a Rayleigh distribution for the fading gain, which can be modeled as

$$
h_{r x, t x}=\sqrt{h_{1}^{2}+h_{2}^{2}},
$$

where $h_{1}$ and $h_{2}$ are two independent Gaussian variables obeying a $N \square\left(0, \delta^{2}\right)$ distribution.

In order to guarantee the QoS requirements for both the victim TV receivers and each D2D link, all channel gains must be continuously estimated for the online optimal power allocation. Exact channel estimation is still a challenge, and this is a topic currently undergoing much research. Fortunately, the deployment of DTV transmitters and TV receivers are static, and the transmission frequencies change relatively infrequently, which makes the channel estimation in TVWS more tractable. Specifically, the channel gains between each D2D link (i.e., $b_{i, j}$ ) can be estimated using pilot-aided channel estimation or other approaches [24]. The channel gains between the transmitters of the D2D links and the victim TV receiver (i.e., $b_{p r, i}$ ) can be estimated by employing sensors near the receiver, and forwarding this information to a central controller [25] (i.e., the geolocation spectrum database). If the channel gains vary slowly over time, the geolocation database can obtain and update the channel gains fast enough to enable the power allocation algorithm to be used. In this paper, we adopt the above signal propagation model as an alternative method of characterizing the propagation environment and quantifying the channel gain of each link.

\subsection{SINR Threshold Model}

As formerly mentioned, the worst-case TV receiver is taken as the victim TV receiver for analysis. It operates on channel $a$, with a central frequency of $f_{a} \cdot p_{t v}^{t x}$ is the transmission power of the DTV transmitter, and the SINR at the TV receiver can then be denoted as

$$
\gamma=\frac{p_{t v}^{t x} b_{r x, t x}^{t v}}{v_{p r}+\sigma_{0}^{2}},
$$


where $\sigma_{0}^{2}$ is the noise power at the TV receiver, $b_{r x, t x}^{t v}=g_{r x, t x}^{t v} h_{r x, t x}^{t v}$ represents the joint gain due to path loss and Raleigh fading between the DTV transmitter and receiver, and $v_{p r}$ is the cumulative interference from the D2D links.

Assuming that there are $N$ D2D links, the channel taken by each link is denoted as $\mathbf{x}=\left\{x_{i}\right\}, 1 \leq i \leq N$, with frequency $f_{x_{i}}$, and all channels have the same bandwidth as the DTV receiver. Due to imperfections in the receiver filter characteristics, interference can occur from either a D2D link on the same channel or adjacent channels. According to previous studies [26], the required SINR threshold for successful signal reception in a TV receiver, with interference on channel $x_{k}$ is defined as

$$
\frac{p_{t v}^{t x} b_{r x, t x}^{t v}}{v_{p}^{x_{k}}+\sigma_{0}^{2}} \geq \gamma(\Delta f),
$$

where $v_{p}^{x_{k}}$ denotes the interference received on channel $x_{k}$ by the TV receiver, and $\Delta f=\left|f_{a}-f_{x_{k}}\right|$ is the frequency offset between the two channels. Fig. 3 gives the SINR protection threshold of each adjacent channel according to Ofcom reference data [27] and measurement results by $[26,27]$. The co-channel refers to $\Delta f=0$, which has the highest protection threshold of all the channels.

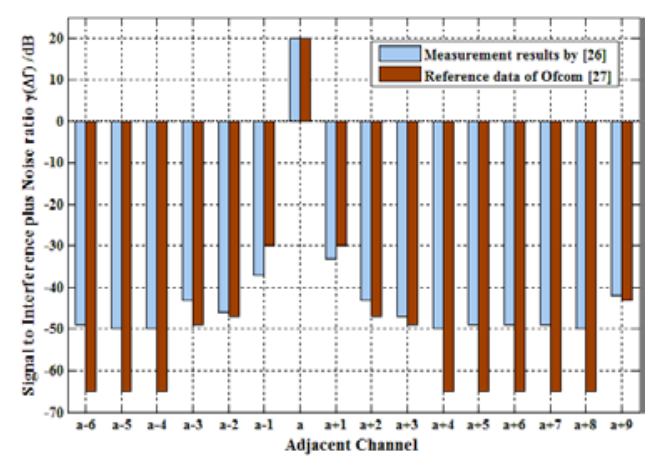

Fig. 3. Protection threshold of SINR on co-channel and adjacent channel

Each active D2D link $i$ imposes the following interference to the TV receiver:

$$
v_{p r, i}=p_{i} b_{p r, i}, i \in\{1, \ldots, N\},
$$

where $p_{i}$ is the transmission power of link $i$, and $b_{p r, i}=\vartheta\left(x_{i}, a\right) g_{p r, i} h_{p r, i} \cdot g_{p r, i} h_{p r, i}$ is the joint attenuation due to path loss and fading, while $\vartheta\left(x_{i}, a\right)$ is the defined adjacent channel interference coefficient, when $x_{i}=a, \vartheta\left(x_{i}, a\right)=1$, otherwise $\vartheta\left(x_{i}, a\right)=\gamma(\Delta f) / \gamma(0)$. Taking all active D2D links within the area of interest into account, the cumulative effect of interference from multiple channels can be modeled as an equivalent co-channel interference by the weighted summation of the interference from each different channel [28]

$$
v_{p r}=\sum_{i=1}^{N} v_{p r, i}=\sum_{i=1}^{N} p_{i} b_{p r, i} .
$$


Then the signal-to-interference-plus-noise ratio at the TV receiver should meet the following constraint:

$$
\gamma=\frac{p_{t v}^{t x} b_{r x, t x}^{t v}}{\sum_{i=1}^{N} p_{i} b_{p r, i}+\sigma_{0}^{2}} \geq \gamma^{t h},
$$

$\gamma^{\text {th }}$ is the threshold level of the SINR at the TV receiver and in the case above, the DTV signal will be received correctly.

Similarly, for the D2D link $i$, the interference from the D2D link $j$ is $v_{i, j}=p_{i} b_{i, j}$, where $b_{i, j}=\vartheta\left(x_{i}, x_{j}\right) g_{i, j} h_{i, j}$ when $i \neq j$, and $b_{i, j}=g_{i, j} h_{i, j}$ otherwise. Hence the aggregate interference due to other links received by $i$ is $v_{i}=\sum_{j \neq i}^{N} v_{i, j}=\sum_{j \neq i}^{N} p_{j} b_{i, j}$. At the receiver of the D2D link $i$, the SINR should not be below the threshold level $\lambda_{i}^{\text {th }}$ for the coexistence of multiple D2D links.

$$
\lambda_{i}=\frac{p_{i} b_{i, i}}{\sum_{j \neq i}^{N} p_{j} b_{i, j}+\sigma_{i}^{2}} \geq \lambda_{i}^{\text {th }} \quad, i \in\{1, \ldots, N\} .
$$

\section{Feasibility Analysis and Problem Formulation}

In this section, we first discuss the feasibility of the system model, and then formulate the problem of interest. Finally, we describe the technical challenges in solving the optimization problem.

\subsection{Feasibility Analysis}

Define an $N \times N$ matrix $\mathbf{M}$ with the $(i, j)$ - th elements as $m_{i, j}=\frac{\lambda_{i}^{\text {th }} b_{i, j}}{b_{i, i}}$ for $i \neq j$ and $m_{i, j}=0$ for $i=j$. In addition, define a column vector $\boldsymbol{\eta}=\left\{\frac{\lambda_{i}^{\text {th }} \delta_{i}^{2}}{b_{i, i}}\right\}$ of length $N$. $\mathbf{p}=\left[p_{1}, p_{2}, \ldots, p_{N}\right]^{T}$ denotes the transmission power across all links if the SINR level at each link's receiver reaches the corresponding threshold, thus

$$
\mathbf{J p} \succ \boldsymbol{\eta},
$$

where $\mathbf{J}=\mathbf{I}-\mathbf{M}$, I refers to the $N \times N$ identity matrix and “ $\succ$ ” is used to denote a component-wise inequality. Introducing positive slack variables $\mathbf{q}=\left[p_{N+1}, p_{N+2}, \ldots, p_{2 N}\right]^{T}$, the inequality (14) can be written as

$$
(\mathbf{J},-\mathbf{I})\left(\begin{array}{l}
\mathbf{p} \\
\mathbf{q}
\end{array}\right)=\boldsymbol{\eta} \text {. }
$$

We assert that the system is strictly feasible if and only if $\mathbf{p}^{\dagger}=\mathbf{J}^{-1}(\boldsymbol{\eta}+\mathbf{q})$ satisfies the following constraint 


$$
\left\{\begin{array}{l}
0<\mathbf{p}^{\dagger} \leq \mathbf{p}^{\max } \\
\mathbf{b}_{\mathrm{pr}} \mathbf{p}^{\dagger}<\frac{p_{t v}^{t x} b_{r x, t x}^{t v}}{\gamma^{t h}}-\sigma_{0}^{2}
\end{array},\right.
$$

in which the column vector $\mathbf{b}_{\mathrm{pr}}=\left\{b_{p r, i}\right\}$, and $\mathbf{p}^{\max }=\left\{p_{i}^{\max }\right\}$ denotes the maximum transmission power level at each transmitter. Intuitively, the total system throughput can only possibly be enhanced when the coexistent communication includes coexistence between the TV receiver and D2D links, as well as guaranteed self-coexistence between the D2D links.

\subsection{Problem Formulation}

In this paper, we use the total throughput as the performance measure of the secondary D2D link system. Thus, the optimization problem is formulated as

$$
\begin{aligned}
& o p t^{*}=\max _{\mathbf{p}=\left\{p_{i}\right\}} \sum_{i=1}^{N} \log _{2}\left(1+\frac{p_{i} b_{i, i}}{\sum_{j \neq i}^{N} p_{j} b_{i, j}+\sigma_{i}^{2}}\right) \\
& \text { s.t. } \quad \mathbf{b}_{\mathrm{pr}} \mathbf{p} \leq \frac{p_{t v}^{t x} b_{r x, t x}^{t v}}{\gamma^{t h}}-\sigma_{0}^{2}, \\
& \quad \frac{p_{i} b_{i, i}}{\sum_{j \neq i}^{N} p_{j} b_{i, j}+\sigma_{i}^{2}} \geq \lambda_{i}^{\text {th }}, \forall i \in\{1, \ldots, N\} .
\end{aligned}
$$

The problem aims to find the optimal power allocation $\mathbf{p}^{*}$ that will maximize the total throughput. The constraints include the condition of protection of the victim TV receiver and the other conditions guarantee the coexistence of multiple D2D links. However, the optimization problem in this form can be very hard to solve, since the objective function is nonlinear and not even convex, and this is the real technical challenge of this problem. Furthermore, the above multivariable optimization problem can be rather difficult and highly dependent on the number of variables. If there are intersecting relationships between multiple variables, numerical results in $[29,30]$ show that huge numbers of iterations are required to obtain the optimum solution for this type of problem. We will later introduce a feasible solution to convert this difficult problem into a more tractable problem and obtain an optimum solution within an acceptable number of iterations.

In the following sections, we focus on making the optimization problem more manageable using some mathematical transformations, and design a polynomial complexity algorithm to find the optimum solution. Note that the constraints (12b) and (12c) are not linear, however an equivalent transformation can be used to give the following linear form,

$$
\begin{aligned}
& f_{1}(\mathbf{p})=\sum_{i=1}^{N} p_{i} b_{p r, i}-\frac{p_{t v}^{t x} b_{r x, t x}^{t v}}{\gamma^{t h}}+\sigma_{0}^{2} \leq 0, \\
& f_{i+1}(\mathbf{p})=-p_{i} b_{i, i}+\lambda_{i}^{t h} \sum_{j \neq i}^{N} p_{j} b_{i, j}+\lambda_{i}^{\text {th }} \sigma_{i}^{2} \leq 0, \forall i \in\{1, \ldots, N\} .
\end{aligned}
$$


Alternatively, the objective function (12a) can be rewritten as the following difference between two convex functions

$$
\sum_{i=1}^{N} \log _{2}\left(1+\frac{p_{i} b_{i, i}}{v_{i}+\sigma_{i}^{2}}\right)=u(\mathbf{p})-w(\mathbf{p})
$$

where both $u(\mathbf{p})$ and $w(\mathbf{p})$ are concave functions

$$
\left\{\begin{array}{l}
u(\mathbf{p})=\sum_{i=1}^{N} \log _{2}\left(\sum_{j=1}^{N} p_{j} b_{i, j}+\sigma_{i}^{2}\right) \\
w(\mathbf{p})=\sum_{i=1}^{N} \log _{2}\left(\sum_{j \neq i}^{N} p_{j} b_{i, j}+\sigma_{i}^{2}\right)^{2}
\end{array} .\right.
$$

The gradient of $w(\mathbf{p})$ at each $p_{i}$ is given by

$$
\frac{\partial w(\mathbf{p})}{\partial p_{i}}=\sum_{j \neq i}^{N} \frac{1}{\ln 2} \frac{b_{j, i}}{v_{j}+\sigma_{j}^{2}}=\sum_{j=1}^{N} \frac{1}{v_{j}+\sigma_{j}^{2}} e_{j, i},
$$

where $e_{j, i}=\left\{\begin{array}{l}0, j=i \\ \frac{b_{j, i}}{\ln 2}, j \neq i\end{array}\right.$, and we denote the column vector $\nabla w(\mathbf{p})=\left\{\tau_{i}\right\}=\left\{\frac{\partial w(\mathbf{p})}{\partial p_{i}}\right\}$. Given a sequence $\left\{p_{i}^{k}\right\}$ of feasible solutions, we can approximate $w(\mathbf{p})$ by its first order Taylor expansion $w\left(\mathbf{p}^{(k)}\right)+\left\langle\nabla w\left(\mathbf{p}^{(k)}\right), \mathbf{p}-\mathbf{p}^{(k)}\right\rangle$ within the neighborhood of $\left\{p_{i}^{k}\right\}$, and then replace the objective function by

$$
-f_{0}(\mathbf{p})=u(\mathbf{p})-w\left(\mathbf{p}^{(k)}\right)-\left\langle\nabla w\left(\mathbf{p}^{(k)}\right), \mathbf{p}-\mathbf{p}^{(k)}\right\rangle,
$$

where $\langle x, y\rangle=x^{T} y$. Since $w(\mathbf{p})$ is concave, $w(\mathbf{p}) \leq w\left(\mathbf{p}^{(k)}\right)+\left\langle\nabla w\left(\mathbf{p}^{(k)}\right), \mathbf{p}-\mathbf{p}^{(k)}\right\rangle$, if $\left\{p_{i}^{k+1}\right\} \quad$ is feasible and $-f_{0}\left(\mathbf{p}^{(k+1)}\right) \geq-f_{0}\left(\mathbf{p}^{(k)}\right)$. We also have $u\left(\mathbf{p}^{(k+1)}\right)-w\left(\mathbf{p}^{(k+1)}\right) \geq-f_{0}\left(\mathbf{p}^{(k+1)}\right) \geq u\left(\mathbf{p}^{(k)}\right)-w\left(\mathbf{p}^{(k)}\right)$, therefore $-f_{0}(\mathbf{p})$ provides a good approximate lower bound for (14), so maximization of function (17) is equivalent to maximization of the original objective function. It is easy to find that each entry in the Hessian matrix $\nabla^{2} f_{0}(\mathbf{p})$ is nonnegative, hence $f_{0}(\mathbf{p})$ is convex. We can then convert (12) into the following optimization problem for each $\left\{p_{i}^{k}\right\}$

$$
\begin{array}{ll}
\min & f_{0}(\mathbf{p}) \\
\text { s.t. } & (13 \mathrm{~b}),(13 \mathrm{c}) .
\end{array}
$$

Equation (18) above is a standard convex optimization programming problem [31], which is more tractable than the original form. 


\section{Algorithm Design and Analysis}

\subsection{Existence of an Optimal Solution}

Before designing an efficient algorithm, we firstly analyze the duality properties to show the existence of an optimum solution and introduce the interior method to solve the problem.

Firstly, by introducing nonnegative variables $\boldsymbol{\kappa}=\left[\kappa_{1}, \ldots, \kappa_{N+1}\right]$ for the inequality in (13b) and (13c), we obtain the Lagrange function as

$$
L(P, \kappa)=f_{0}(\mathbf{p})+\sum_{i=1}^{N+1} \kappa_{i} f_{i}(\mathbf{p}) .
$$

According to [31], the dual function is defined as an unconstrained minimization of the Lagrangian, and the constrained optimization (18) can now be solved via the unconstrained optimization problem

$$
g(\mathbf{\kappa})=\inf _{\mathbf{p}} \quad L(\mathbf{p}, \mathbf{\kappa})
$$

The optimal value of the Lagrange dual problem is denoted as

$$
d^{*}=\underset{\kappa \geq 0}{\operatorname{maximize}} g(\mathbf{\kappa}),
$$

We refer to the difference $o p t^{*}-d^{*}$ as the optimal duality gap of the primal problem, which is zero when strong duality holds [32]. In particular, the primal problem (18) is convex, and Slater's condition can be satisfied due to the limits in (10) and (11): there exists any valid $\left\{p_{i}^{k}\right\}$ such that

$$
\begin{aligned}
& \sum_{i=1}^{N} p_{i}^{k} b_{p r, i}-\frac{p_{t v}^{t x} b_{r x, t x}^{t v}}{\gamma^{t h}}+\sigma_{0}^{2}<0, \\
& -p_{i}^{k} b_{i, i}+\lambda_{i}^{\text {th }} \sum_{j \neq i}^{N} p_{i}^{k} b_{i, j}+\lambda_{i}^{\text {th }} \sigma_{i}^{2}<0, \forall i \in\{1, \ldots, N\} .
\end{aligned}
$$

where the inequality constraints must hold with strict inequality. Since the convexity and Slater's theorem states that strong duality holds, this meets the following important theorem [31]: If a convex optimization problem with differentiable objective and constraint functions satisfies Slater's condition, then the Karush-Kuhn-Tucker (KKT) conditions provide necessary and sufficient conditions for optimality.

$$
\nabla f_{0}\left(\mathbf{p}^{*}\right)+\sum_{i=1}^{N+1} \kappa_{i}^{*} \nabla f_{i}\left(\mathbf{p}^{*}\right)=0
$$

The above equation is just one of the KKT conditions known as the recall function(19). According to the above theorem, the optimal duality gap is zero and the dual optimum can be attained when $\mathbf{p}^{*}$ is optimal and, together with $\boldsymbol{\kappa}^{*}$, satisfies the KKT conditions. However, for the formulated problem in this paper, it is impossible to solve the KKT conditions analytically. Therefore, we must turn to interior-point methods for solving the problem (18) and the KKT conditions.

\subsection{Optimum Power Allocation for Throughput Maximization Algorithm}

So far, the intractable nonconvex optimization problem (12) has been well approximated by the convex optimization problem(18). Most importantly, we have analyzed the existence of an 
optimal solution and obtained the optimality conditions (KKT conditions). In this section, this paper attempts to develop a quick iterative algorithm. Base on the previous analysis, we introduce the barrier method to locate the global optimal solution, which is a representative technique from the class of interior point methods. The motivation is that the barrier method is simple to implement and shows good performance. The barrier method includes the Newton method for unconstrained minimization and a logarithmic barrier function. A few other methods should also be named. One is the efficient solver of the active set method, which differs from interior point methods since no barrier term is used to ensure that the algorithm remains interior with respect to the inequality constraints [33]. Another is the representative genetic algorithm (GA) of intelligent algorithms. GA is an adaptive heuristic search algorithm that solves the optimization problem based on evolutionary ideas of natural selection and genetics [34]. To verify its effectiveness, the proposed algorithm is compared with these two popular methods and this is discussed further with the simulation results.

First, we consider the following unconstrained approximation of the inequality constrained problem (18)

$$
\min f_{0}(\mathbf{p})+\frac{1}{t} \phi(\mathbf{p}), t>0 .
$$

where $\phi(\mathbf{p})=-\sum_{i=1}^{N+1} \log \left(-f_{i}(\mathbf{p})\right)$ is called the logarithmic barrier function of (18), since $-(1 / t) \log (-u)$ is differentiable, convex and increases with $u<0$. We can use Newton's method to search for the minimum of (24) within $\mathbf{p} \in\left[0, \mathbf{p}^{\max }\right]$. The reason why we choose Newton's method is that it exhibits the fastest convergence rates by using second derivation information for the computation of the descent direction. As [32] suggests, the quality of approximation improves as parameter $t$ grows.

For $t>0$, we assume $\mathbf{p}^{*}(t)$ is the solution of (24), which is strictly feasible, satisfies

$$
f_{i}\left(\mathbf{p}^{*}(t)\right)<0, \quad i \in\{1, \ldots, N+1\},
$$

and the first derivation condition

$$
0=\nabla f_{0}\left(\mathbf{p}^{*}(t)\right)+\frac{1}{t} \nabla \phi\left(\mathbf{p}^{*}(t)\right)=\nabla f_{0}\left(\mathbf{p}^{*}(t)\right)+\sum_{i=1}^{N+1} \frac{1}{t} \frac{1}{-f_{i}\left(\mathbf{p}^{*}(t)\right)} \nabla f_{i}\left(\mathbf{p}^{*}(t)\right),
$$

Defining $\kappa_{i}^{*}(t)=-\frac{1}{t^{*} f_{i}\left(\mathbf{p}^{*}(t)\right)}, \quad i \in\{1, \ldots, N+1\}$, then (26) is expressed as

$$
\nabla f_{0}\left(\mathbf{p}^{*}(t)\right)+\sum_{i=1}^{N+1} \kappa_{i}^{*}(t) \nabla f_{i}\left(\mathbf{p}^{*}(t)\right)=0,
$$

Compared with equation (23) of the KKT conditions, we can see that $\mathbf{p}^{*}(t)$ minimizes teh Lagrangian $L(P, \kappa)$, therefore we have

$$
\begin{aligned}
g\left(\kappa^{*}(t)\right) & =L\left(\mathbf{p}^{*}(t), \kappa^{*}(t)\right)=f_{0}\left(\mathbf{p}^{*}(t)\right)+\sum_{i=1}^{N+1} \kappa_{i}^{*}(t) f_{i}\left(\mathbf{p}^{*}(t)\right) \\
& =f_{0}\left(\mathbf{p}^{*}(t)\right)+\sum_{i=1}^{N+1}-\frac{1}{t}=f_{0}\left(\mathbf{p}^{*}(t)\right)-\frac{N+1}{t}
\end{aligned}
$$


It can be seen that the optimal duality gap is less than $\frac{N+1}{t}$, thus $\mathbf{p}^{*}(t)$ is no more than $\frac{N+1}{t}$ suboptimal and $\mathbf{p}^{*}(t)$ converges to optimal as $t$ tends to infinity.

With the former analysis, we can use the following iterative algorithm to reach the optimum value of (12).

Algorithm: Optimum power allocation for throughput maximization

initialize: Judge whether the feasible solution conditions of (10) and (11) can be satisfied, if so, initialize $k=0, \mathbf{p}^{(0)}=\left\{p_{i}^{0}\right\}, t=t^{(0)}>0, \mu>1$ and tolerance $\varepsilon>0$; otherwise, the optimal solution does not exist.

repeat

1. set $f_{0}(\mathbf{p})=-\left(u(\mathbf{p})-w\left(\mathbf{p}^{(k)}\right)-\left\langle\nabla w\left(\mathbf{p}^{(k)}\right), \mathbf{p}-\mathbf{p}^{(k)}\right\rangle\right)$.

\section{2. repeat}

1). start at $\mathbf{p}^{(k)}$, utilize Newton's method to compute $\mathbf{p}^{*}(t)$ by minimizing $f_{0}(\mathbf{p})+\frac{1}{t} \phi(\mathbf{p})$.

2). update $\mathbf{p}^{(k)}=\mathbf{p}^{*}(t)$.

3). increase $t, t=\mu t$.

until $(N+1) / t^{(0)} \leq \varepsilon$.

3. update $\mathbf{p}^{(k+1)}=\left\{\max \left(0, \min \left(p_{i}^{*}, p_{i}^{\max }\right)\right)\right\}$.

4. $k=k+1$,

until $\left|f_{0}\left(\mathbf{p}^{(k)}\right)-f_{0}\left(\mathbf{p}^{(k-1)}\right)\right| \leq \varepsilon$

\subsection{Implementation details}

This algorithm should be embedded in the geolocation database, since that database is designed to hold the overall information. Generally, after finishing the channel coordination and admission control process in [20], the feasible solution condition of (10) and (11) are satisfied, and the formulated problem has an optimal solution. Firstly, given the initial point $\mathbf{p}^{(0)}=\left\{p_{i}^{0}\right\}$, at its neighborhood, we can get the first order Taylor derivation of the original objective function, and we can solve the approximate convex problem instead. The convex problem is then further transformed into an unconstrained problem, and Newton's method can be used to find the solution.

The algorithm contains two-tier iterations (strictly there are three but we treat the barrier method, which utilizes Newton's search, as a single iteration and analyze them integrally). The barrier method is the inner iteration and the objective function update is the outer iteration. Equation (28) suggests that the solution of (24) is no more than $(N+1) / t$ suboptimal with respect to the solution of (18). Therefore this restriction provides a stopping criterion for the barrier method, which guarantees that we have an $\varepsilon$ suboptimal solution. Then the obtained solution will be regarded as the new feasible starting point for the updated objective function. 
The whole iterative process terminates after several outer iterations at $\left|f_{0}\left(\mathbf{p}^{(k)}\right)-f_{0}\left(\mathbf{p}^{(k-1)}\right)\right| \leq \varepsilon$ when no further gain in the solution can be achieved within the tolerance threshold $\varepsilon$.

\subsection{Algorithm Initialization}

To perform the above algorithm, the initialization requires a start point $\mathbf{p}^{(0)}$, which must be strictly feasible, and can be obtained by solving (10) and (11). Usually feasible points are not unique, and many methods can perform this search. One method used is to perform linear programming as

$$
\begin{array}{ll}
\min & \tau \\
\text { s.t. } & f_{i}(\mathbf{p}) \leq \tau, \quad i \in\{1, \ldots, N+1\}, \\
& 0<p_{i} \leq p_{i}^{\max }, i \in\{1, \ldots, N\} .
\end{array}
$$

where any valid $\left\{p_{i}^{0}\right\}$ can be taken as the start point of the above problem, and the initial value of $\tau$ can be set as $\tau^{(0)}=\max _{i=1, \ldots, N+1} f_{i}\left(\mathbf{p}^{(0)}\right)$. Once $\tau<0$, a strictly feasible starting point can be found, if $\tau \geq 0$, the system is not strictly feasible. We do not provide details on this calculation for a more concise description, and just give the feasible start points for the algorithm.

The other question is how to set $t^{(0)}$ and $\mu$. For typical applications of the barrier method, $t$ is increased by a constant factor $\mu>1$ (typically 10) until the minimization of (24) by Newton's method reaches a sufficient level of accuracy [33]. For the convergence rate of the barrier method in the proposed algorithm, $t^{(0)}$ should be neither too small nor too large. One reasonable method is to choose a value of $t^{(0)}$ which gives a similar order of magnitude of $(N+1) / t^{(0)}$ and $f_{0}\left(\mathbf{p}^{(0)}\right)-o p t^{*}$.

\subsection{Complexity Analysis}

The highest computational cost of the proposed algorithm lies with the barrier method. It should be mentioned that, with Newton's method as the unconstrained optimization solver, the computational complexity of the inner iteration is dominated by the solution of the linear equality system $\nabla^{2} f(x) \square \Delta x_{n t}=-\nabla f(x)$ for the Newton step $\Delta x_{n t}$ with a given $\nabla^{2} f(x)$ and $\nabla f(x)$ [33]. The generic methods for solving $\Delta x_{n t}$ require computational operations of the approximate order $O\left(N^{3}\right)$; however, the computation cost can be further reduced by the structure of $\nabla^{2} f(x)$. Since $\nabla^{2} f(x)$ is a symmetric and positive defined matrix, this offers computational savings of around half of the generic case [31]. The convergence of the barrier method can be directly analyzed and after $k$ inner iterations, the duality gap is $(N+1) /\left(\mu^{k} t^{(0)}\right)$, so the desired accuracy $\varepsilon$ is achieved after 


$$
n_{\text {inner }}=\frac{\log \left((N+1) /\left(\varepsilon t^{(0)}\right)\right)}{\log (\mu)}
$$

inner iterations. In fact, the outer iteration converges very quickly, and it only takes several iterations, denoted as $n_{\text {outer }}$, as long as the dimensions of the problem are not too large. Denoting the complexity of Newton's method as $C_{1}$, then the total complexity of the proposed algorithm is

$$
C_{\text {Algorithm }}=n_{\text {outer }} n_{\text {inner }} C_{1} .
$$

In summary, by exploiting the feasible solution for the original optimization problem and proposed algorithm, the huge computational requirements to find the optimum solution can be reduced to polynomial complexity.

\section{Simulation and Experimental Evaluation}

\subsection{Simulation Setup}

As the scenario depicted in Section II, multiple D2D links can spatially reuse the TV channel licensed to a DTV system. The AP passes the communication requests and evacuation information received in a period to the geolocation database. The geolocation database has detailed information about registered D2D links, so can use an algorithm to allocate the regulated transmission powers to the new group of D2D links. For real-time purposes, the algorithm computational rate should as fast as possible. In Table 1, we list some of the system parameters used in the following simulations, for which the parameters of the DTV system and D2D communications are based on the specification in [22, 23, 35].

We consider a single cell network in the simulation, where D2D pairs are uniformly distributed in the cell. The average distance between transmitter and receiver for each D2D pair is $20 \mathrm{~m}$, and the cell center is $500 \mathrm{~m}$ from the victim TV receiver. There are two examples given below. The first example explains how the proposed algorithm can adapt to diverse hardware configurations and shows the convergence and accuracy performance; the second example deals with a large-dimensional problem.

Table 1. System parameters used in simulations

\begin{tabular}{ccc}
\hline Parameter & Value & Comments \\
\hline \hline$f_{a}$ & $6 \mathrm{MHz}$ & Bandwidth of a channel \\
\hline$p_{t v}^{t x}$ & $700 \mathrm{MHz}$ & Transmission power of DTV transmitter \\
\hline$\sigma_{i}^{2}, 0 \leq i \leq N$ & $90 \mathrm{dBm}$ & Noise power \\
\hline$\left(x_{0}, y_{0}\right)$ & $-130 \mathrm{dBm}$ & 2-D coordinates of the victim TV receiver \\
\hline$d_{r x, t x}^{t v}$ & $(0,0)$ & Radius of DTV protection region \\
\hline$\alpha$ & $150 \mathrm{~km}$ & Path loss exponent \\
\hline$l_{T}^{T V}$ & 2.5 & Height of DTV transmitter \\
\hline$l_{T}, l_{R}, l_{R}^{T V}$ & $100 \mathrm{~m}$ & Antenna height of WSD, TV receiver \\
\hline
\end{tabular}




\subsection{Simulation results}

Example 1: For the first investigation of the proposed method, a small number of D2D links are considered where all links are working on the same channel as the victim TV receiver. The parameters are set as follows: Number of links $N=5$, SINR threshold of TV receiver $\gamma^{\text {th }}=20 \mathrm{dBm}$, and the maximum transmission power of the D2D links and SINR threshold of each receiver are randomly set as $\mathbf{p}^{\max }=[20,22,25,23,25]^{T} \mathrm{dBm}$ and $\lambda^{\text {th }}=[19,15,21,15,18]^{T} \quad$ dB. With a feasible starting point of $\mathbf{p}^{(0)}=\left[\begin{array}{ll}11.8820 & 2.04189 .25232 .84387 .0279\end{array}\right]^{T}$ dBm, Fig. 4 shows that the algorithm converges to the optimal solution after approximately eight outer iterations, which is very efficient. Since the transmission power of each D2D link varies, the SINR level at the D2D receivers and the victim TV receiver are adjusted accordingly. In Fig. 5, the solid lines refer to the adjustment tendency of each SINR level, and the dotted lines are the diverse SINR thresholds with respect to the different QoS constraints of each receiver. It can be seen that the proposed algorithm coordinates well with the D2D links without any violation of the QoS requirements. We set the tolerance threshold $\varepsilon=10^{-4}$ to guarantee a reasonable accuracy for the solution, while avoiding too many iterative cycles for further tiny gains.

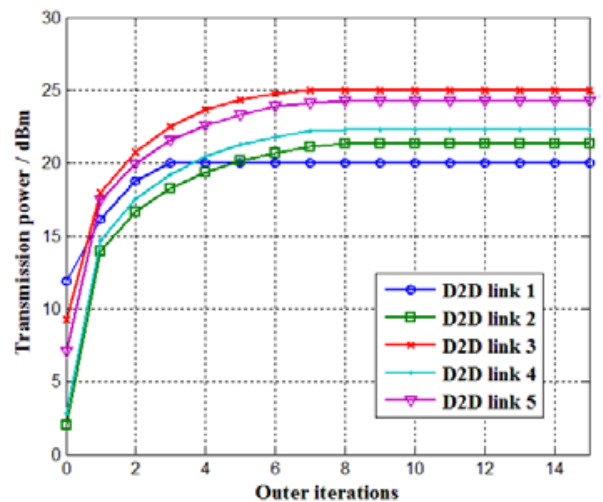

Fig. 4. Convergence of transmission power

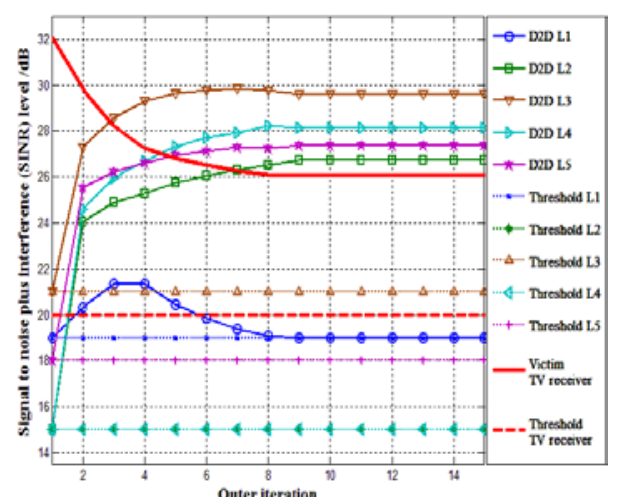

Fig. 5. The adjustment of SINR levels

Fig. 6 shows a snapshot of the inner iteration, which traces the minimization process by Newton's method. In the proposed method, we solve (18) instead of (12), so we are concerned principally with the accuracy of the approximate derivation. As shown in Fig. 7, $-f_{0}(\mathbf{p})$ approximates to a lower bound of $u(\mathbf{p})-w(\mathbf{p})$, and updates itself after each iteration, which enables the approximation gap to be reduced notably and finally reach a high approximate accuracy.

Example 2: In this example, we test the proposed method for a larger dimension scenario containing three available channels $(a, a+1, a+2)$ with each channel shared by ten D2D links. Both the co-channel and adjacent channel interference are considered in this simulation. The maximum transmission power levels of the mobile users/devices are set as $20 \mathrm{dBm}$ and the SINR threshold of the receivers are $20 \mathrm{~dB}$. The performance of each of the different methods is depicted in Fig. 8. In this figure, point 1 and point 2 are two different initial feasible points for the proposed algorithm and the active set algorithm. As can be seen, our methods can also 
work well for a large-dimensional power allocation problem, although given different initial points the proposed method can track the optimum value with only several outer iterations, because the proposed method is actually a method to find the optimal feasible point. The active set method also shows strong performance, but the results are not as optimized as the proposed algorithm. Additionally, there may be a gap when the active set method is initialized from different feasible points. The reason is that each Newton search step in the proposed method requires second order information (gradients and Hessians), whereas the active set method uses only first order information (gradient). Hence the proposed algorithm can perform better, particularly near the optimum value. Since the search result of the genetic algorithm may be uncertain each time, we repeat the computational process several times and select the best one from historical results. Compared with the previous two methods, the results of the genetic algorithm still have much room for improvement. There are other conclusions that can be drawn from Fig. 8, including that although the outer iteration does not require much increased computation, the inner iteration needs more iterations to converge, which can also be inferred by (30). When the proposed algorithm converges, the transmission power of all the D2D links can be determined; consequently, the SINR level at each receiver is stabilized at its optimal state from an overall perspective (see Fig. 9).

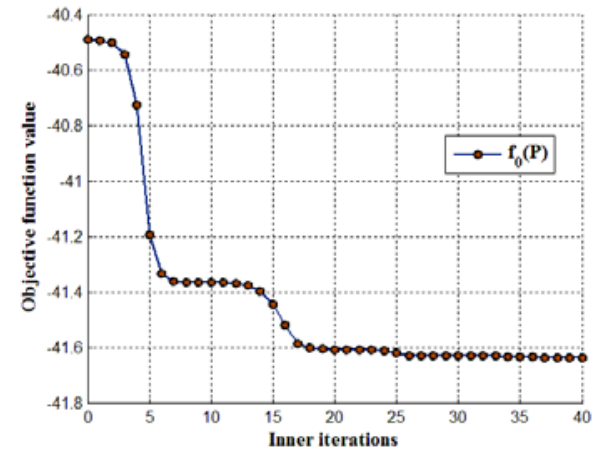

Fig. 6. Convergence of objective function

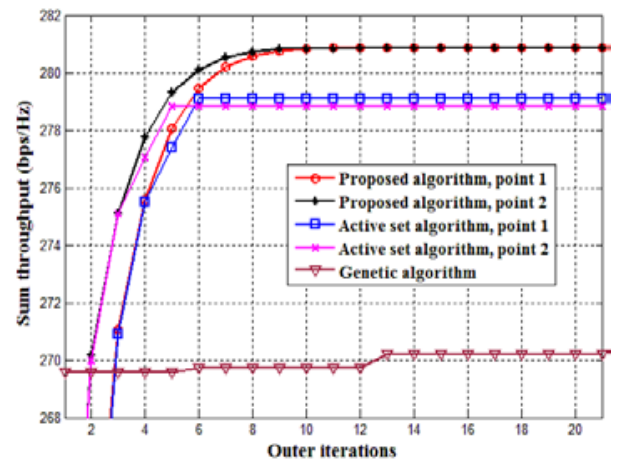

Fig. 8. Performance comparison

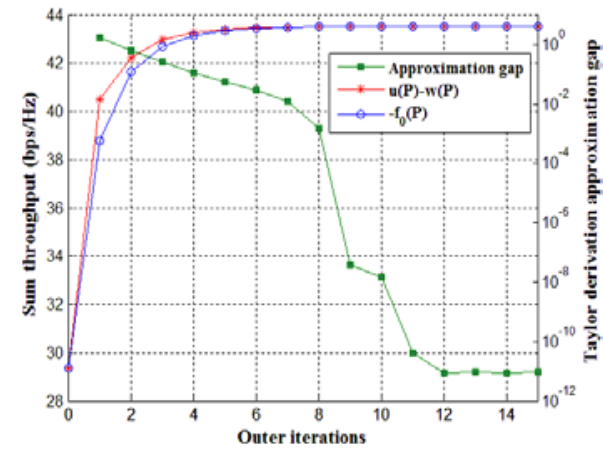

Fig. 7. Convergence of sum throughput

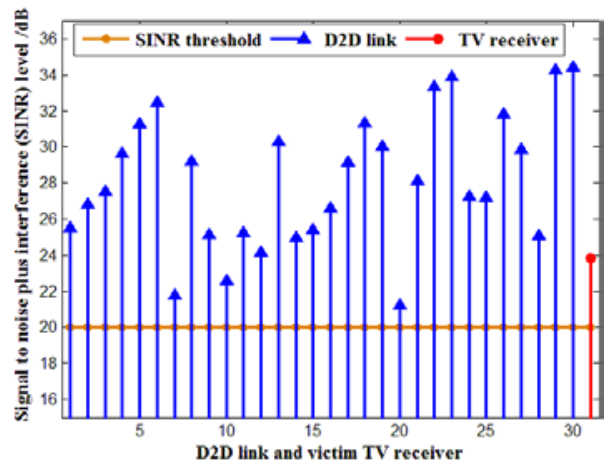

Fig. 9. Optimal SINR level vs. threshold SINR level

\section{Conclusion}

In this paper, we have proposed a geolocation database assisted power allocation method to address multiple device-to-device communications within TV white space. Firstly, we have introduced a scenario where D2D communications access TVWS, and all the D2D links 
distributed within a cell are spatially reusing incumbent DTV channels, which gives rise to the important challenge of coexistence between the TV receivers and the D2D links as well as self-coexistence between multiple D2D links. Secondly, we have formulated a sum throughput oriented optimization problem with the coexistence conditions as constraints. However, the original problem is neither linear nor convex; hence, it is difficult to find a solution. Then we have used an efficient method to convert the problem into a series of more tractable convex problems. We then have introduced the interior point method to solve the problem and have designed an effective algorithm based on the barrier method to find the solution with a low computational cost of polynomial complexity. Simulations show that the proposed method works well either in small-dimensional or large-dimensional problem, and has sufficient adaptability to adjust parameters for diverse devices. Furthermore, the proposed algorithm has better performance compared with the popular active set algorithm and genetic algorithm.

\section{References}

[1] G. Staple and K. Werbach, "The end of spectrum scarcity [spectrum allocation and utilization]," Spectrum, IEEE. 48-52, 2004. Article (CrossRef Link).

[2] M. Fitch, M. Nekovee, S. Kawade, K. Briggs, and R. MacKenzie, "Wireless service provision in TV white space with cognitive radio technology: A telecom operator's perspective and experience," Communications Magazine, IEEE, vol. 49, pp. 64-73, 2011. Article (CrossRef Link).

[3] FCC, "Second Memorandum Opinion and Order In the Matter of Unlicensed Operation in the TV Broadcast Bands, Additional Spectrum for Unlicensed Devices Below $900 \mathrm{MHz}$ and in the $3 \mathrm{GHz}$ Band," September, 2010. Article (CrossRef Link).

[4] R. Murty, R. Chandra, T. Moscibroda, and P. Bahl, "Senseless: A database-driven white spaces network," Mobile Computing, IEEE Transactions on, vol. 11, pp. 189-203, 2012. Article (CrossRef Link).

[5] E. CEPT, "Technical and operational requirements for the possible operation of cognitive radio systems in the 'White Spaces' of the frequency band 470-790 MHz," 2011.

Article (CrossRef Link).

[6] L. Shi, K. W. Sung, and J. Zander, "Controlling aggregate interference under adjacent channel interference constraint in TV white space," in Proc. of Cognitive Radio Oriented Wireless Networks and Communications (CROWNCOM), 2012 7th International ICST Conference on, pp. 1-6, 2012. Article (CrossRef Link).

[7] J. Wang, G. Ding, Q. Wu, L. Shen, and F. Song, "Spatial-temporal spectrum hole discovery: a hybrid spectrum sensing and geolocation database framework," Chinese Science Bulletin, vol. 59, pp. 1896-1902, 2014. Article (CrossRef Link).

[8] D. Feng, L. Lu, Y. Wu, G. Li, S. Li, and G. Feng, "Device-to-device communications in cellular networks," Communications Magazine, IEEE, vol. 52, pp. 49-55, 2014. Article (CrossRef Link).

[9] W. Lili, R. Q. Hu, Q. Yi, and W. Geng, "Enable device-to-device communications underlaying cellular networks: challenges and research aspects," Communications Magazine, IEEE. 90-96, 2014. Article (CrossRef Link).

[10] H. Shi, R. V. Prasad, V. S. Rao, I. G. Niemegeers, and M. Xu, "Spectrum-and energy-efficient D2DWRAN," Communications Magazine, IEEE, vol. 52, pp. 38-45, 2014. Article (CrossRef Link).

[11] M. Jo, T. Maksymyuk, B. Strykhalyuk, and C.-H. Cho, "Device-to-device-based heterogeneous radio access network architecture for mobile cloud computing," IEEE Wireless Communications, vol. 22, pp. 50-58, 2015. Article (CrossRef Link).

[12] X. Lin, J. Andrews, A. Ghosh, and R. Ratasuk, "An overview of 3GPP device-to-device proximity services," Communications Magazine, IEEE, vol. 52, pp. 40-48, 2014. Article (CrossRef Link). 
[13] C.-H. Yu, K. Doppler, C. B. Ribeiro, and O. Tirkkonen, "Resource sharing optimization for device-to-device communication underlaying cellular networks," Wireless Communications, IEEE Transactions on, vol. 10, pp. 2752-2763, 2011. Article (CrossRef Link).

[14] B. Kaufman and B. Aazhang, "Cellular networks with an overlaid device to device network," in Proc. of Signals, Systems and Computers, 2008 42nd Asilomar Conference on, pp. 1537-1541, 2008. Article (CrossRef Link).

[15] H. Min, J. Lee, S. Park, and D. Hong, "Capacity enhancement using an interference limited area for device-to-device uplink underlaying cellular networks," Wireless Communications, IEEE Transactions on, vol. 10, pp. 3995-4000, 2011. Article (CrossRef Link).

[16] D. Feng, L. Lu, Y. Wu, G. Y. Li, G. Feng, and S. Li, "Device-to-Device Communications Underlaying Cellular Networks," Communications, IEEE Transactions on, vol. 61, pp. 3541-3551, 2013. Article (CrossRef Link).

[17] G. Ding, J. Wang, Q. Wu, Y.-D. Yao, F. Song, and T. Tsiftsis, "Cellular-Base-Station Assisted Device-to-Device Communications in TV White Space," Selected Areas in Communications, IEEE Journal on, pp. 1-1, 2015. Article (CrossRef Link).

[18] G. P. Villardi, Y. D. Alemseged, C. Sun, C.-S. Sum, T. H. Nguyen, T. Baykas, et al., "Enabling coexistence of multiple cognitive networks in TV white space," Wireless Communications, IEEE, vol. 18, pp. 32-40, 2011. Article (CrossRef Link).

[19] K. Doppler, M. Rinne, C. Wijting, C. B. Ribeiro, and K. Hugl, "Device-to-device communication as an underlay to LTE-advanced networks," Communications Magazine, IEEE, vol. 47, pp. 42-49, 2009. Article (CrossRef Link).

[20] Z. Xue and L. Wang, "Geolocation database based resource sharing among multiple device-to-device links in TV white space," in Proc. of 7th International Conference on Wireless Communications and Signal Processing (WCSP 2015), 2015. Article (CrossRef Link).

[21] L. B. Le and E. Hossain, "Resource allocation for spectrum underlay in cognitive radio networks," Wireless Communications, IEEE Transactions on, vol. 7, pp. 5306-5315, 2008. Article (CrossRef Link).

[22] T. S. Rappaport, Wireless communications: principles and practice, Second ed. vol. 2: prentice hall PTR New Jersey, 2002. Article (CrossRef Link).

[23] J. Wang, S. Filin, T. Baykas, M. A. Rahman, C. Song, and H. Harada, "A feasible neighbor discovery algorithm for coexistence control system over TVWS," in Proc. of Wireless Communications and Networking Conference (WCNC), 2012 IEEE, pp. 3244-3248, 2012. Article (CrossRef Link).

[24] G. Chen, X.-H. Yu, and J. Wang, "Adaptive channel estimation and dedicated pilot power adjustment based on the fading-rate measurement for a pilot-aided CDMA system," Selected Areas in Communications, IEEE Journal on, vol. 19, pp. 132-140, 2001. Article (CrossRef Link).

[25] T. Banerjee, C. Ghosh, and D. P. Agrawal, "Wireless sensor based dynamic channel selection in cellular communication by cognitive radio approach," in Proc. of Cognitive Radio Oriented Wireless Networks and Communications, 2006. 1st International Conference on, pp. 1-5, 2006. Article (CrossRef Link).

[26] E. Obregon, L. Shi, J. Ferrer, and J. Zander, "A model for aggregate adjacent channel interference in TV white space," in Proc. of Vehicular Technology Conference (VTC Spring), 2011 IEEE 73rd, pp. 1-5, 2011. Article (CrossRef Link).

[27] Ofcom, "Digital Dividend: Cognitive Access Statement on License Exempting Cognitive Devices Using Inter-leaved Spectrum," July 2009. Article (CrossRef Link).

[28] L. Shi, K. W. Sung, and J. Zander, "Secondary spectrum access in TV-bands with combined co-channel and adjacent channel interference constraints," in Proc. of Dynamic Spectrum Access Networks (DYSPAN), 2012 IEEE International Symposium on, pp. 452-460, 2012. Article (CrossRef Link).

[29] N. Vucic, S. Shi, and M. Schubert, "DC programming approach for resource allocation in wireless networks," in Proc. of Modeling and Optimization in Mobile, Ad Hoc and Wireless Networks (WiOpt), the 8th International Symposium on, pp. 380-386, 2010. Article (CrossRef Link). 
[30] J. Huang, R. A. Berry, and M. L. Honig, "Distributed interference compensation for wireless networks," Selected Areas in Communications, IEEE Journal on, vol. 24, pp. 1074-1084, 2006. Article (CrossRef Link).

[31] B. Stephen and V. Lieven, Convex optimization: Cambridge university press, 2009. Article (CrossRef Link).

[32] H. Hindi, "A tutorial on convex optimization II: duality and interior point methods," in Proc. of American Control Conference, pp. 686-696, June 2006. Article (CrossRef Link).

[33] S. Mishra, U. Topcu, and M. Tomizuka, "Optimization-based constrained iterative learning control," Control Systems Technology, IEEE Transactions on, vol. 19, pp. 1613-1621, 2011. Article (CrossRef Link).

[34] Introduction to Genetic Algorithms: Article (CrossRef Link).

[35] G. Stuber, S. M. Almalfouh, and D. Sale, "Interference analysis of TV-band whitespace," in Proc. of the IEEE, pp. 741-754, 2009. Article (CrossRef Link). 


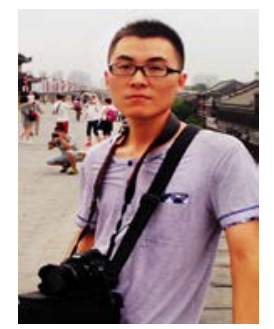

Zhen Xue received his B.S. degree in electrical engineering from Xidian University, Xi'an, China, in 2012, and his M.S. degree in communications and information systems in College of Communications Engineering, Nanjing, China, in 2015. He is currently pursuing the Ph.D. degree in communications and information system in Institute of Communications Engineering, PLA University of Science and Technology. His research interests are wireless communications and cognitive radio networks.

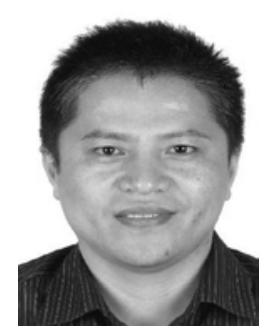

Liang Shen received his B.S. degree in Communications Engineering and M.S. degree in Communications and Information System from the Institute of Communications Engineering, Nanjing, China, in 1988 and 1991 respectively. He is currently a professor at the PLA University of Science and Technology, China. His current research interests are information theory and digital signal processing and wireless networking.

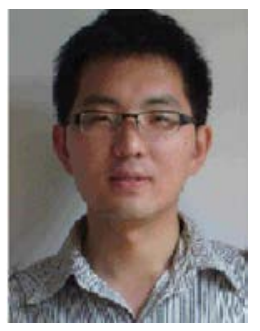

Guoru Ding received his B.S. degree in electrical engineering from Xidian University, Xi'an, China, in 2008 and his Ph.D. degree in communications and information systems in College of Communications Engineering, Nanjing, China, in 2014. Since 2014, he has been an assistant professor in College of Communications Engineering, and also a research fellow in National High Frequency Communications Research Center of China. Since April 2015, he has been a Postdoctoral Research Associate at the National Mobile Communications Research Laboratory, Southeast University, Nanjing, China. His current research interests include Massive MIMO, Cognitive Radio Networks, Wireless Security, Statistical Learning, and Big Spectrum Data Analytics for 5G Wireless Networks. He currently serves as an Editor of KSII Transactions on Internet and Information Systems, and an invited reviewer for 10+ Journals such as IEEE Signal Processing Magazine, IEEE Communications Magazine, IEEE Transactions on Signal Processing, IEEE Transactions on Communications, and IEEE Transactions on Wireless Communications, etc. He was a recipient of Best Paper Awards from IEEE VTC2014-Fall and IEEE WCSP 2009. He is a IEEE/ACM/CCF member, the Secretary of IEEE 1900.6 Standard Association Working Group, and also a voting member of IEEE 1900.6.

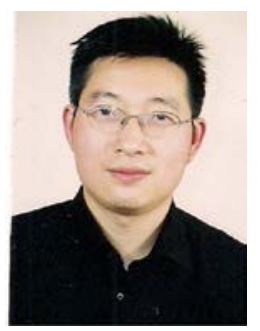

Qihui Wu received his B.S. degree in communications engineering, M.S. degree and Ph.D. degree in communications and information systems from Institute of Communications Engineering, Nanjing, China, in 1994, 1997 and 2000, respectively. From 2003 to 2005, he was a Postdoctoral Research Associate at Southeast University, Nanjing, China. From 2005 to 2007, he was an Associate Professor with the Institute of Communications Engineering, PLA University of Science and Technology, Nanjing, China, where he is currently a Full Professor. From March 2011 to September 2011, he was an Advanced Visiting Scholar in Stevens Institute of Technology, Hoboken, USA. Dr. Wu's current research interests span the areas of wireless communications and statistical signal processing, with emphasis on system design of software defined radio, cognitive radio, and smart radio. 